des plus grandes lignes actuelles de transport d'énergie, comparable en tous points à celles construites en Amérique. L'exécution de tels travaux est très rapide et fait honneur au secteur qui l'a entreprise.

L'utilisation des forces du Rhin amènera bientôt la construction de lignes semblables dans l'inlérèt même de tousl es Français. Les secteurs devront inaugurer une politique nouvelle de contrats et de vente d'énergie pour la répandre à profusion, et il est à souhaiter que des groupements de secteurs se fassent, comme cela a ėté tenté en Suisse (Voir article de M.de Watteville, dans la $H . B$. de mars-avril $1922, \mathrm{n}^{\circ} 13$ ), pour jouer le rôle de chef de distribution en ajustant les puissances des différences centrales créées, aux variations de consommation dans une journée et suivant les régions.

Il est aussi à souhaiter que les capitaux engagés et les efforts faits pour construire de tels transports le soient suivant un plan général d'ensemble, dont chacun pourrait tirer le meilleur profit.

\title{
Méthode thermométrique de Mesure de Rendement des Turbines hydrauliques.
}

\section{Précautions à prendre pour l'Application correcte de cette Méthode.}

\author{
Par E. POIRSON, Ingénieu" électricien I. E. G.
}

La méthode thermométrique do mesure du rendement des lurbines, qui a fait l'objet de nombreuses publications dans les revues techniques et qui, notamment dans La Houille Blanche de septembre-octobre 1920 et de novembre-décembre 1921, a donné matière à des publications de résultats très complets, suppose, comme on le sait, l'exécution de quelques mesures très simples, au moins théoriquement, des prises de température de l'eau à la sortie des turbines, à leur entrée, et cnfin de l'eau détendue soutirée de la conduite forcée.

Cette méthode, connue déjà sous le nom de Méthode de MM. Barbillion et Poirson, ses deux auteurs, a fait l'objet de nombreuses applications dans des usines de chûtes diverses. On peut dire que, tant que la chûte reste supérieure à $40 \mathrm{~m}$. environ, la méthode s'applique avec une réelle facilité et dans des conditions lout à fait remarquables de précision.

Cependant, il importe, pour que cette précision soit effective, qu'un certain nombre de précautions soient prises, qui ne peuvent être indiquées que comme le fruit d'une longue expérience. Ayant eu personnellement à appliquer la méthode dans le plus grand nombre de cas, au cours d'expériences faites dans les usines de Sociétés dont nous avions le contrôle, nous avons cru bon de résumer ci-après l'ensemble de la doctrine opératoire à observer croyant ainsi rendre service à nos collègues expérimentateurs dans la matière. En particulier, nous citerons les expériences très intéressantes, faites aux usines de Fully (Suisse), sous la direction de M. Boucher. La chûte de Fully est actuellement la chûte la plus haute équipée dans le monde $(1.631 \mathrm{~m}$.). Les résultats trouvés par les ingénièurs de la Société exploitant la chûte de Fully ont été de tous points comparables à ceux que nous avons obtenus nous-mêmes au cours de nombreuses expériences.

La formule : $r_{0}=\frac{427\left(\Theta_{2}^{\prime}-\Theta_{2}\right)}{H}$ donne le rendement avec exactitude, sous la réserve que :

$\Theta_{2}=$ Température de l'eau sortant de la turbine dans le canal de fuite,

$\Theta_{0}^{\prime}=$ Température de l'eau. détendue dans le récipient,

$\mathrm{H}^{2}=$ Hauteur manométrique,

soient mesurées correctement, et il est utile de donner à cet égard les indications pratiques suivantes :

Les causes d'erreur principales sont : a) Variations diurnes ou autres de la température $(-)$, de l'eau arrivant à la turbine,

b) Dans la mesure de $\Theta_{y}^{\prime}$, soutirage de l'eau en un mauvais endroit de la conduite forcée, ou débit insuffisant de l'eau soutirée.

c) Dans la mesure de $\boldsymbol{H}_{2}$, influence d'une trop grande capacité, relativement au débit, du canal de fuile en amont du point où l'on relève $(\rightarrow$,

d) En hiver, pour les températures voisines de $0^{\circ}$, influence de glaçons en suspension dans l'eau, et faussanl l'échauffement de l'eau,

e) Erreurs de mesures thermométriques et manométriques,

f) Mesures trop hâtives des températures avant la stabilisation des régimes.

Voici à cet égard les précautions élémentaires à prendre:

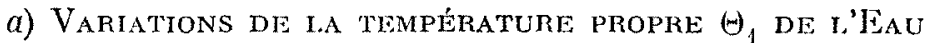

$1^{\circ}$ Variations diurnes. - Il est un fait connu dans les régions montagneuses surtout, c'est que la température des eaux suit les variations diurnes de la tempćrature ambiante, c'est-àdire varie d'une façon continue et relativement lente. Il y a chaque jour un maximum et un minimum de température.

Les variations peuvent atteindre $1^{\circ}$. à $2^{\circ}$ par heure, soit $0^{\circ}, 017$ à 00,034 par minute, ce qui est appréciable lorsque les inesures demandent quelques minutes et portent sur des différences à mesurer de $0^{\circ}, 100$ à $1^{\circ}$, généralement.

$2^{\circ}$ Coups de soleil. - En deuxième lieu, les alternances de soleil et d'ombre causées par les nuages, sur le canal d'amenée ou les conduites forcées, se traduisent immédiatement par des variations correspondantes de la température de l'eau. Celles-ci sont plus rapides que les diurnes et il faut éviter d'opérer en pareilles circonstances où il est plus difficile de saisir les températures instantanées.

$3^{\circ}$ Arrêts. - Enfin, forsque une usine a été arrêtée un certain temps, l'eau en quantité importante arrêtée dans les conduites et le canal d'amenée prend la température ambiante. Lorsque on remet en marche, cette eau met un certain temps pour s'écouler, et lorsque Iui succède l'eau vive du cours d'eau, il se produit à un certain moment une rapide variation de température. Il faut éviter, là encore, d'effectuer les mesures dans ces instants là : il faut attenđ̆re le temps suffisant que le régime stable soit établi. 
Correction des Variations de Température propre DE L'EAU

Un peut prendre simultanément les températures:

$$
\begin{aligned}
& \Theta_{2}^{\prime} \text { (récipient de détente) } \\
& \text { et } \Theta_{2} \text { (canal de fuite) }
\end{aligned}
$$

avec deux thermomètres soigneusement étalonnés, l'un par rapport à l'autre ; mais il peut y avoir des erreurs appréciables de ce fait.

Il paraît plus sûr d'opérer comme suit :

On mesure $\left(\Theta_{0}^{\prime}\right.$ à l'instant $t_{1}$, soit $\left[()_{0}^{\prime}\right]_{t_{1}}$

On mesure $\Theta_{2}$ à l'instant $t_{2}$, soit $\left[\Theta_{Q}\right]_{l_{2}}$

On mesure $\theta_{2}^{\prime}$ à l'instant $t_{3}$, soit $\left.\left[\Theta_{2}^{\prime}\right]\right]_{l,}$

Dans l'intervalle $t_{3}-t_{1}, \theta_{2}^{\prime}$ a varié de :

$$
\left[\Theta_{2}^{\prime}\right]_{3}-\left[\Theta_{2}^{\prime}\right]_{t_{1}}
$$

Ces variations sont proportionnelles à l'étendue des intervalles correspondants.

On peut donc dire qu'à l'instant $t_{2}$, où l'on mesurait $\Theta_{2}$, la vraie température $\Theta_{0}^{\prime}$ dans le récipient de détente, était :

$$
\Theta_{2}^{\prime}=\left[\Theta_{2}^{\prime}\right]_{l_{1}}+\left(\left[\Theta_{2}^{\prime} l_{l_{3}}-\left[\Theta_{2}\right]_{t_{1}}\right) \times \frac{t_{2}-t_{1}}{l^{3}-t_{1}}\right.
$$

le terme correctif pouvant d'ailleurs être positif ou négatif, suivant que la température propre est ascendante ou descendante.

En opérant ainsi avec le même thermomètre on évite des erreurs de mesure.

Comme le triple relevé ci-dessus ne demande généralement que 4 à 5 minutes, on peut le recommencer plusieurs fois jusqu'à ce que l'on obtienne la stabilité des résultats, malgré les variations possibles de température propre de l'eau.

On peut, d'ailleurs, si on le désire, opérer à des instants de la journée où la lempérature propre de l'eau ne varie pas (maximum ou minimum de la variation diurne), mais cela n'est même pás nécessaire, grâce à la simple correction ci-dessus indiquée, dont il faut souligner toute l'importance.

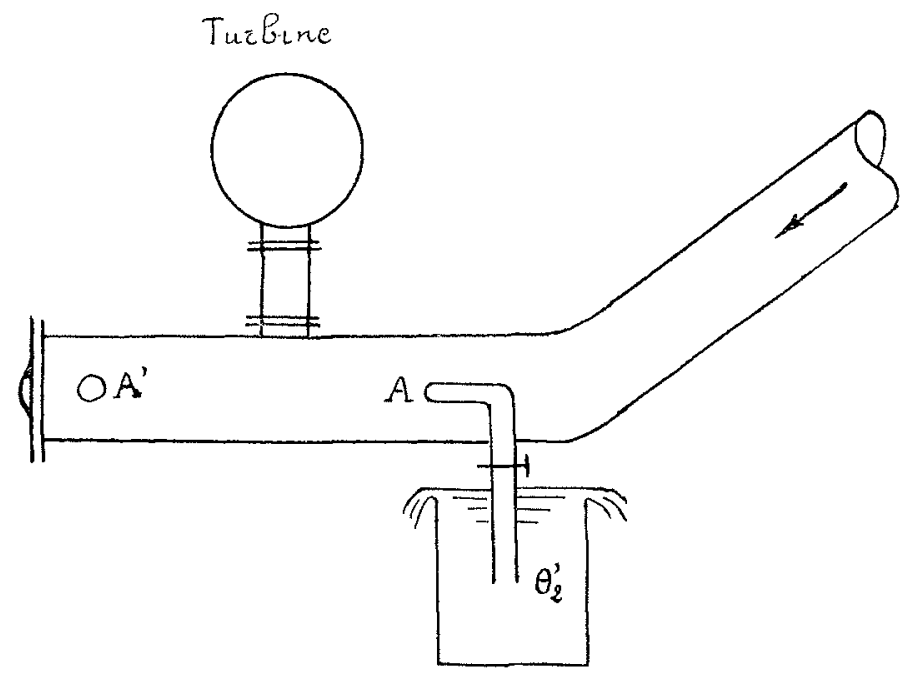

b) Erreurs de Mesure sur $\left(-y_{2}^{\prime}\right.$

10 Point de soutirage.-L'eau détendue dont on veut mesurer la température doit ètre bien celle qui est en mouvement pour arriver à la turbine et non de l'eau immobilisée en quelque point A' des tuyauteries où il n'y a pas de circulation, et où l'eau peut avoir une température quelconque, différente de celle de l'eau qui travaille.

On soutirera done en un point tel que $A$, si possible sur la tubulure d'alimentation de la turbine, ou bien sur la conduite, en amont de celle-ci et proche d'elle.

$2^{\circ}$ Tuyau de soutirage. - Le soutirage peut se faire correctement de différentes manières :

- Sur un petit tuyau de branchement de manomètre.

- Sur petit robinet de vidange ou autre, etc..

Il faut que le tuyau de soutirage soit aussi court que possible pour qu'il ne puisse y avoir réchauffement sensible par l'ambiance, A ce point de vue, le débit d'eau soutirée doit être assez fort, relativement à la masse des tuyauteries, robinet, récipient de soutirage. Pour ce dernier un seau de 10 à 15 litres suffit, avec un débit de 'l'ordre, de 1 litre par seconde; il faut que l'eau s'échappe autant que possible sans vitesse de ce récipient.

Il faut d'ailleurs attendre un temps suffisant pour que le seau lui-même ait bien pris la température de l'eau, et le réservoir du thermomètre doit être placé dans l'eau rapprochée du jet qui afflue; on y trouve à peu près la même température que dans le jet lui-même, généralement, car c'est dans le robinet que l'eau travaille presque totalement, si la vitesse du jet est assez laible. L'idéal serait que le robinet où s'échauffe l'eau plonge lui-même dans l'eau du récipient, pour échapper à l'ambiance, mais on a reconnu que cela n'est pas indispensable.

\section{c) Erreurs de Mesure sur $\Theta_{Q}$}

L'eau sortant de la turbine dont on s'occupe ne doit évidemment pas être mélangée à d'autres eaux, ce qui fausserait gros. sièrement la température $\theta_{2}$.

On doit s'assurer en particulier :

$1^{\circ}$ Qu'on ne rejette dans le canal de fuite, en amont du premier point où l'on relève $\left(\omega_{2}\right.$, aucune eau de services auxiliaires de refroidissement, etc...;

$2^{\circ}$ Que les robinets vannes des autres turbines ne perdent pas une quantité d'eau notable relativement au débit de la turbine considérée.

Toutefois, quand on peut accéder directement au débouché de la turbine dans le canal de fuite, on échappe à ces causes d'erreur.

On peut mesurer la température en plaçant directement le thermomètre dans l'eau du canal, ce qui oblige souvent à une gymnastique appropriée, pour faire des mesures correctes. On peut aussi simplement, à l'aide d'un seau au bout d'une corde, prélever de l'cau du canal et y mesurer de suite sa température ; dans ce cas, le seau doit avoir trempé quelques minutes au préalable dans l'eau du canal, on rejette l'eau qu'il contient alors, et on le remplit rapidement à nouveau au moment de laire la mesure.

\section{Capacité du Canal de Fuite}

Si le canal de fuite sous les turbines, en amont du point où l'on relève $\Theta_{a}$, a une trop grande capacité relativement au débit de la turbine considérée, il y a une cause d'erreur importante, parfois même grossière du fait des mélanges. On y échappe si l'on peut prendre $\Theta_{2}$ tout près de la turbine considérée ; sinon la méthode peut être inapplicable dans un tel cas particulier.

Mais un tel cas est assez rare.

\section{d) TEMPÉRATURES VOISINES DE $0^{\circ}$}

Quand la température de l'eau est voisine de $0^{\circ}$, c'est-à-dire que l'eau charrie de la neige ou des glaçons, on conçoit immédiatement qu'il ne faut pas appliquer cette méthode, parce que la présence des glaçons en suspension dans l'eau empêcherail 
l'échauffement théorique, à cause de leur chaleur de fusion absorbant les calories.

Ne pas appliquer la méthode en pareil cas.

\section{e) Erreurs de Mesures}

10 Thermométriques. - II faut attendre que le thermomètre ail bien pris la température, ce qui, avec les thermomètres spéciaux employés donnant 2 à $8 \mathrm{c} / \mathrm{m}$ par degré, demande 1 minute $1 / 2$ environ ; donner de légers chocs à l'ongle pour s'assurer que la colonne de mercure a bien pris sa place.

Il faut aussi lire $\theta_{2}^{\prime}$ et $A_{2}$, avec le thermomètre vertical (il est étalonné ainsi), ou tout au moins incliné également dans les mesures (influence de la pression de la colonne de mercure dilalant plus ou moins le réservoir du thermomètre).

Il est commode de disposer d'un jeu de thermomètres courts et sensibles :

$$
\begin{aligned}
& \text { L'un gradué de } 0^{\circ} \text { à } \mathrm{T}_{1} \\
& \text { L'autre de } \mathrm{T}_{1}-2^{\circ} \text { à } \mathrm{T}_{2} \\
& \text { L'autre de } \mathrm{T}_{2}-2^{\circ} \text { à } \mathrm{T}_{3}
\end{aligned}
$$

et ainsi de suite.

Il faut vérifier qu'il n'y a pas d'interruption dans la colonne de mercure.

$2^{\circ}$ Manométriques. - Si l'on n'a pas confiance dans les mano mètres de l'usine, il est facile de brancher un manomètre étalon, exact à moins de $1 \%$ près.

On connaît bien généralement la hauteur de chute totale de l'usine, donc la pression statique nette que doit indiquer le manomètre quand il n'y a pas de débit, ce qui est un moyen de le vérifier.

La lecture de la pression manométrique a autant d'importance que celle des températures. Si l'on n'avait qu'un mano- mètre douteux il serait préférable de se baser sur la hauteur de chute réelle connue par nivellement à la conslruction de l'usine, et de calculer les pertes de charge dans la conduite forcée, pour le débit évalué approximativement.

Enfin, les mesures donnant le rendement étant très rapidts, on peut les recommencer plusieurs fois pour avoir une certitude plus grande sur les résultats.

f) Stabilisation des régimes. - Il est enfin utile de revenir sur la nécessité avant de faire la série de mesures thermométriques correspondant à un régime donné, d'attendre un temps suffisant pour la stabilisation des différentes températures des pièces el des parois. 5 à 10 minutes suffisent généralement pour cela, si les débits d'eau en jeu sont suffisamment forts par rapport à l'ambiance.

Telles sont les principales précautions indiquées par l'expérience déjà acquise.

En résumé, l'application correcte de la formule :

$$
\eta=\frac{427\left(\Theta_{2}^{\prime}-\Theta_{2}\right)}{H}
$$

nécessite :

- La mesure correcte de la température de détente $\Theta^{\prime}{ }_{Q}$ de l'eau affluant à la turbine.

- La mesure correcte de la température $\Theta_{0}$ de l'eau s'échappant au même instant de la turbine.

- La mesure correcte de la pression manométrique $\mathrm{H}$ existant à cet instant sur la turbine.

Le degré d'approximation sera celui résultant de ces trois mesures.

Il pourra souvent être à moins de $1 \%$ près.

\title{
Sur l'utilisation de très petites sources d'énergie hydraulique pour les besoins de l'Agriculture.
}

\author{
Par L. RIGOTARD, Iàgénieur Agronome d̀ Chad̀tesse (Isère)
}

Depuis un cértain temps on envisage de plus en plus l'emploi de l'énergie mécanique pour exécuter les travaux agricoles. C'est une application d'une incontestable utilité pour notre production nationale ; cependant la mise au point de cette adaptation de l'énergie hydraulique ou électrique apparaît ardue dans la pratique. On peut ici rappeler pour mémoire qu'il est assez difficile de fournir du courant électrique à des abonnés utilisant la force d'une façon très irrégulière et dans bien des cas pendant un très pelit nombre d'heures par an. D'autre part, il est gênant et onéreux, pour des raisons assez nombreuses de divers ordres, pour une grande ligne de transport d'énergie, d'effectuer' en cours de route des distributions aux petites agglomérations rurales, très nombreuses et de peu d'importance moyenne,

Il semble que l'on pourrait envisager l'utilisation sur une base très étendue des nombreux petits cours d'eau qui sillonnent nos campagnes, pour fournir sur place à ces populations rurales l'énergie que la rareté de main-d'œuvre leur fait une obligation d'utiliser.

Le grand obstacle à cette utilisation de la houille blanche des très petits cours d'eau réside dans le coût élevé des installations de faible puissance. Or les agriculteurs n'utilisant l'énergie qu'un très petit nombre d'heures par jour ou même, de plus, peu de jours par an, il importerait de pouvoir réaliser des installations dont les frais d'amortissement et d'entretien ne s'éloignent pas trop de l'ordre de grandeur des frais de main-d'œuvre économisés. Dans la plupart des cas il ne s'agirait pas de faire des installations ayant, au point de vue technique, une certaine perfection, mais d'utiliser à peu de frais une partie de l'énergie disponible.

Les agriculteurs ont d'ailleurs l'esprit ainsi fait que dès qu'ils ont reconnu les avantages d'une méthode, ils se lancent d'euxmêmes dans l'exagération de son emploi. Si donc on amène un certain nombre d'entre eux à faire des installations qui leur donnent satisfaction sous le rapport de la commodité comme sous le rapport financier, on peut être sûr de voir se développer rapidement l'utilisation de la houille blanche dans les campagnes. Il existe dans les communes rurales sur une partie importante du territoire de la France une quantité de houille blanche suffisante pour l'agriculture qui y est pratiquée. Ainsi dans un groupe de trois communes contiguës du département de l'Isère que nous prenons comme exemple, il existe, à proximité ou même enclavées dans des exploitations agricoles, un certain nombre de chutes d'eau aménageables. Répartie sur quatre ou cinq ruisseaux dont plusieurs débitent environ cent litres à la seconde et pouvant donner facilement des chutes de 20 à 30 mètres, la puissance 